\title{
VoIP-based Intra-village Teleconnectivity: An Architecture and Case Study
}

\author{
Janak Chandarana, K. Sravana Kumar, Srinath Perur, Raghuraman Rangarajan, \\ Sameer Sahasrabuddhe and Sridhar Iyer \\ KReSIT, IIT Bombay \\ Email: \{janak, sravana, srinath, raghu, sameerds, sri\}@it.iitb.ac.in
}

\begin{abstract}
Several solutions exist to bridge the last-mile gap in rural telecom. These extend a point of presence from a town to kiosks in surrounding villages. In this paper, we deal with extending teleconnectivity from these kiosks to various points inside the village. Since laying additional PSTN lines is expensive, we use a single PSTN line connected to a software exchange, and VoIP calls are carried over Ethernet or WiFi. We use soft-phones on computing devices or analog phones with adapters to make or receive calls from different parts of the village. As proofof-concept, we deploy the proposed solution in a rural test-bed and report our experience.
\end{abstract}

\section{INTRODUCTION}

Around $70 \%$ of India's population lives in villages. These villages typically consist of around 250 households and are located between two to three kilometres from each other. There are larger market towns located every 30 to 40 kilometres, which are served by optical fibre backbone. These act as a Point of Presence (PoP) for telecommunication for surrounding villages, with each PoP serving around 250 to 300 villages. The problem of connecting these PoPs to the villages can be regarded as the problem of last mile rural connectivity.

Last mile connectivity: Cellular coverage around the market towns typically extends only for about five kilometres. This is because traditional cellular coverage proves too expensive to connect all villages to their nearest PoP. Income levels for rural India are lower than the national average. Average monthly income in rural India is around INR 2500, and it is estimated that the average household can spend less than INR 100 per month on telecom services. Since Average Revenue Per User (ARPU) from villages is too low to recover infrastructure and service costs, cellular coverage in rural India is negligible at present [1].

The key to Internet and telephony access for rural India is a public kiosk which provides a basket of services. A kiosk can expect one or two voice calls to be made on a continuous basis, and most of a kiosk's income is expected to be from voice traffic. Assuming two kiosks per village, the revenue of each can only be of the order of INR 5000 per month. Most existing initiatives for the last mile focus on extending reach from the PoP to the village kiosk. The Department of Telecommunications (DoT), through its Village Public Telephone (VPT) scheme, aims to have at least one telephone installed in each of approximately six lakh villages identified in the 2001 census [2]. As of August 2005 , VPTs were deployed in $83.3 \%$ of the targeted villages [3]. The next phase involves installing a second telephone in villages with a population over 2000 . Besides DoT and TRAI (Telecom Regulatory Authority of India) initiatives, WLL (Wireless in Local Loop) solutions using corDECT [4], WiFiRe [5] designed to operate in the unlicensed band, and the Digital Gangetic Plain project [6] using multi-hop 802.11 links with directional antennas are recent attempts to address the last mile issue.

Intra-village connectivity: In this paper we address what we term the intra-village connectivity issue for rural connectivity: given a single gateway from a village to an external telecom network, how can we extend the reach further within the village? An acceptable solution would ideally be:

- easy to deploy and maintain;

- using off-the-shelf components;

- low on operational expenditure; and

- low on power consumption.

In this paper we propose an architecture based on WiFi and Ethernet for intra-village connectivity (Section II), and describe a proof of concept deployment study (Section III). While more detailed studies are required to evaluate our architecture, we believe it is particularly suited for use in villages whose spread and population are not large enough for other solutions to be economical. 


\begin{tabular}{|c|c|c|}
\hline Solution & Advantages & Disadvantages \\
\hline $\begin{array}{l}\text { VoIP over } \\
\text { WiFi and Ethernet }\end{array}$ & $\begin{array}{l}\text { Intra-village mobility, cost-effective, } \\
\text { available off-the-shelf }\end{array}$ & Limited wireless range \\
\hline EPBX & $\begin{array}{l}\text { Robust against environmental } \\
\text { conditions, familiar technology }\end{array}$ & $\begin{array}{c}\text { Licensing required, } \\
\text { prohibitive installation cost for small scale deployments }\end{array}$ \\
\hline Cellular & $\begin{array}{l}\text { Mobility, availability of } \\
\text { cheap handsets }\end{array}$ & $\begin{array}{l}\text { High installation costs, low ROI for } \\
\text { operators, poor coverage in } \\
\text { remote areas }\end{array}$ \\
\hline Ethernet based & Reliable, simple & Unrealistic in a rural scenario, cost scales with size \\
\hline
\end{tabular}

TABLE I

COMPARISON AMONG ALTERNATE SOLUTIONS

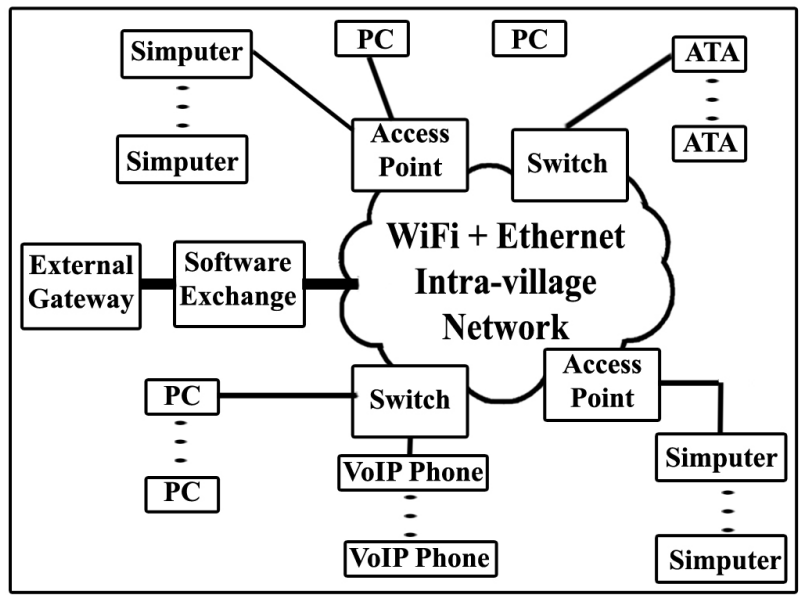

Fig. 1. Intra-village connectivity: architecture

We also believe it is easy to deploy, maintain and replicate in other rural scenarios.

\section{ARChitecture}

Most villages have a limited number of PSTN lines, often only one. Making or receiving calls involves visiting the kiosk, which may not always be convenient. A kiosk also cannot be used for communication within the village. The utilisation of such a line is likely to be suboptimal. Instead of adding more PSTN lines, it may be possible to use the existing line more efficiently by deploying an intra-village network. We aim to extend the coverage of one or a small number of PSTN lines by using an intra-village wireless and Ethernet network.

Our proposed architecture is shown in Figure 1. The external gateway shown is usually a PSTN line, but it can be replaced with any other telecom gateway such as a Cordect, WiFiRe or WLL point of presence. This incoming line interfaces with a software exchange. This software exchange is connected to various communication devices through the proposed intra-village network and carries VoIP calls. This network consists of WiFi Access Points and Ethernet switches deployed to cover the required area of the village.

A caller from the PSTN line uses the exchange to connect to the appropriate end user's device through the intra-village network. Similarly, in the other direction, a caller reaches the exchange through the intra-villagenetwork and connects to the PSTN line. In addition, voice calls between various end devices within the village can be made through the exchange.

The advantages of using such an architecture are:

- increased usage of limited telecom resources by allowing use from multiple locations;

- intra-village connectivity without use of additional resources; and

- use of off-the-shelf components to build the network.

External gateway: The external gateway interfaces a telecom point of presence (PoP) with an IP network. For example, the gateway can be a hardware device which allows VoIP clients in the network to access a PSTN line. This hardware device, called an Analog Telephone Adapter (ATA), converts between analog voice and VoIP, and between signalling protocols. Similar gateways for other technologies can be used to interface them with the network.

Software exchange: A software exchange is a software implementation of a private branch exchange (PBX). The exchange allows VoIP end-user devices in a network to make calls to one another. The exchange is also interfaced with the external gateway, allowing devices to make external calls. Incoming calls from the gateway are also routed through the exchange to the called end-user device. Typically the external gateway and the exchange are co-located. 


\begin{tabular}{|c|c|c|c|c|c|c|}
\hline Location & Distance(metre) & $\begin{array}{l}\text { Comments on LOS } \\
\text { and obstruction }\end{array}$ & Avg. RTT & $\begin{array}{c}\text { Packet } \\
\text { Loss }(\%)\end{array}$ & Signal & SNR \\
\hline Open ground (near kitchen) & 55 & LOS & $35 \mathrm{~ms}$ & 0 & -53 & 42 \\
\hline Kitchen & 150 & partial LOS, some foliage & $36 \mathrm{~ms}$ & 1 & -60 & 40 \\
\hline Kitchen (inside) & 155 & partial LOS, some foliage, wall & $67 \mathrm{~ms}$ & 29 & -77 & 23 \\
\hline Meeting hall & 210 & partial LOS, some foliage & $33 \mathrm{~ms}$ & 12 & -75 & 27 \\
\hline Kitchen (near road) & 170 & NLOS, dense foliage and building & $49 \mathrm{~ms}$ & 30 & -71 & 29 \\
\hline Jeep shed & 180 & NLOS, dense foliage & $121 \mathrm{~ms}$ & 47 & -77 & 23 \\
\hline
\end{tabular}

TABLE II

RF RESULTS

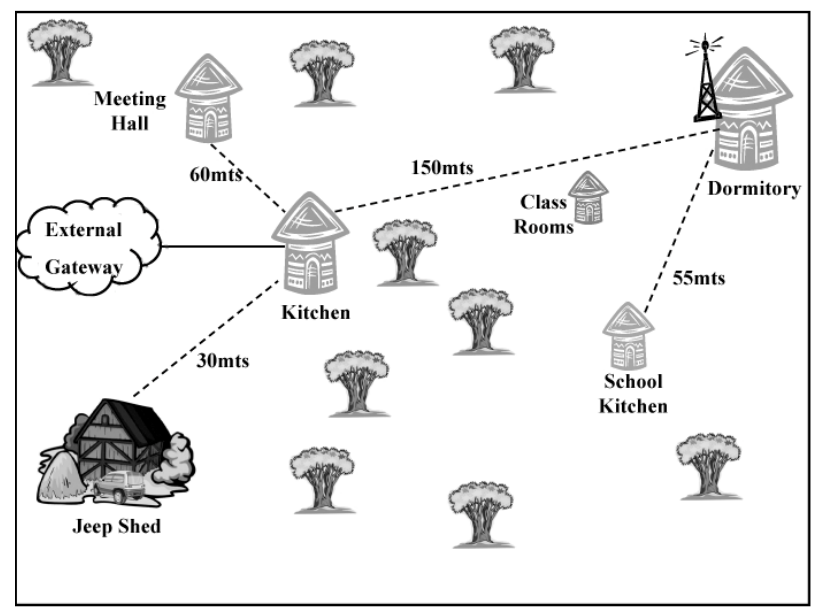

(a) Timbaktu layout

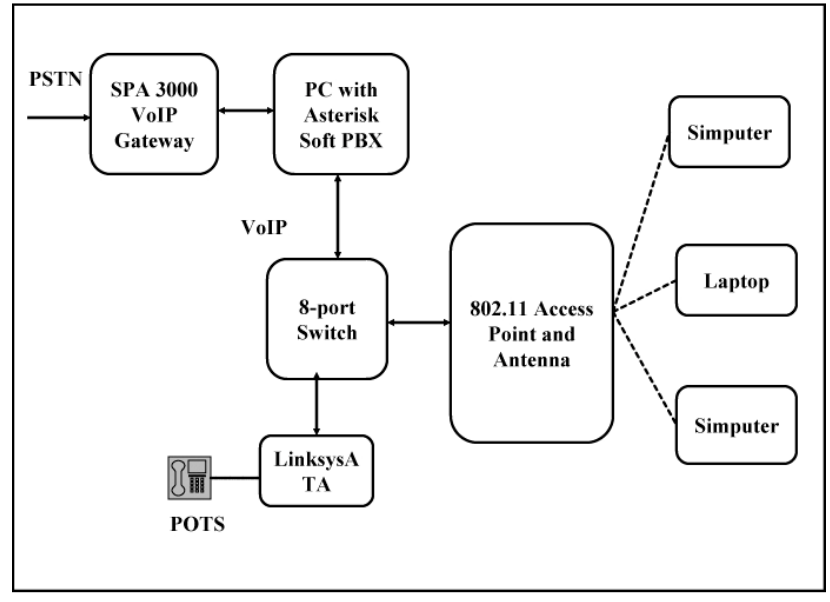

(b) Case study setup

Fig. 2. Proof-of-concept deployment

Intra-village network: The end-user devices located in various parts of the village must be connected to the exchange. For this, we propose a hybrid intra-village network with both wired and wireless elements connected in a mesh topology. This is because a village environment may not allow a homogenous network architecture to be constructed. A wireless network is easy to deploy, and a small number of access points can support a large number of clients, including mobile clients. But the functioning of a wireless network can be constrained by terrain and the presence of obstacles. While a wired network does not suffer from these constraints and allows reliable connectivity, it requires laying of cables for each end-user device, and can be expensive and inconvenient. A wired network may also be constrained by the absence of power points at convenient locations for switches and repeaters. A hybrid design with both wired and wireless elements allows for flexibility in design.

End-user devices: End devices can be wireless handhelds, PCs, or phones. Phones can be VoIP phones or analog phones. However, analog phones need to be attached to suitable adaptors (ATAs [7]) before they can be connected to the network.

Other solutions: There are several possibilities for intra-village connectivity. Traditional solutions such as EPBX, cellular and Ethernet-based networks can be deployed. Table I summarises the advantages and disadvantages of these solutions vis-a-vis a $\mathrm{WiFi}+$ Ethernet deployment.

\section{CASE STUDY}

The Timbaktu Collective is a voluntary organisation working for sustainable development in the drought prone Anantapur district of Andhra Pradesh. There are about a dozen homes, a school, a community kitchen and a few administrative buildings within a one $\mathrm{km}$ radius (Figure 2(a)). Because of its remote location, Timbaktu lacks cellular coverage and its connectivity is through a single PSTN line. This phone is currently located in the community kitchen. The phone sees frequent use(mostly incoming), and each time a user needs to walk to the 


\begin{tabular}{|l|c|c|}
\hline Quality & Packet drop & Delay \\
\hline Very Good & $0 \%$ & $<3 \mathrm{~ms}$ \\
\hline Good & $<5 \%$ & $<10 \mathrm{~ms}$ \\
\hline Average & $10 \%$ & $<20 \mathrm{~ms}$ \\
\hline Poor & $>25 \%$ & $>30 \mathrm{~ms}$ \\
\hline
\end{tabular}

TABLE III

DESCRIPTION OF VOIP CALL QUALITY

kitchen to make or receive a call. The remote location of Timbaktu prevents deployment of additional lines from the nearest switch. It is located in a valley surrounded by hills, and this eliminates cellular coverage as an alternative. Timbaktu thus becomes a good case study for our architecture. However, the area provides a challenging environment for wireless deployment. The collective has dense foliage and lacks an external electricity supply. Solar panels are installed to meet power requirements.

Our aim was to allow residents of Timbaktu to make and receive calls from multiple locations using the single PSTN line. We do this by using existing computers at the school and residences as communication devices. To connect the PSTN line to locations close to the kitchen, we established an Ethernet network to carry VoIP connections. We used hand-held PDAs (Simputers) as mobile clients.

We set up the wireless network as follows:

1) we established location and installed AP and antenna;

2) performed RF measurements;

3) set up the software PBX and interfaced it with the $\mathrm{AP}$

4) set up the analog phone and wireless handhelds; and

5) performed VoIP tests using different instruments.

$A P$ and antenna installation: We used a D-link DWLg2100 AP along with a D-link ANT24-0801 directional wireless antenna with $8.5 \mathrm{dBi}$ gain [8]. The AP was set to operate in $802.11 \mathrm{~b}$ infrastructure mode. The antenna was set up on top of the dormitory building. We performed measurements to evaluate the RF characteristics of the deployment area. We did this by performing ping measurements for various point-to-point links from a single AP, and observing delay and packet loss characteristics. We observed that intervening foliage caused the wireless link to deteriorate. Some observations are shown in Table II. These provided us with a set of candidate locations within reach of the AP, and were chosen locations for performing VoIP test calls.
Software exchange: We chose the Asterisk soft PBX [9] for the following reasons:

- it is open source software;

- it is readily available and widely used;

- it runs on various platforms;

- it needs no additional hardware for VoIP and supports many VoIP protocols; and

- it can inter-operate with almost all standards-based telephony equipment using relatively inexpensive hardware.

The Asterisk server was set up on a Intel laptop with a wireless card running Fedora core 3. Installation and configuration details are given in [10].

PSTN gateway: The PSTN line requires an interface to communicate with the software exchange. This PSTN gateway allows calls to be made or received from a landline to any user on the network. Such gateways, called Analog Telephone Adaptors (ATAs), bridge analog telephony with IP networks. We use the Sipura SPA3000 telephone adaptor [11] for this purpose. Sipura allows a single landline to be interfaced with the software exchange. It also has a port for connecting existing analog phones.

Communication devices: An analog phone connection was provided using a LinkSys phone adaptor (ATA PAP2 [7]). The adaptor allows the use of the phone in a VoIP-enabled network. The adaptor has two ports for analog phones and an Ethernet jack to interface with a network. It supports the Session Initiation Protocol (SIP [12]) and multiple voice compression techniques. It can be configured either through the analog telephone via voice prompts or through a web-based configuration (the adaptor has a built-in web server). The adaptor was connected directly to the AP through the Ethernet port. The adaptor setup and configuration details are given in [13]. We also set up the Asterisk server to translate numbers dialed from the phone into SIP usernames. An example dial plan is given in [10].

As our WiFi handheld we chose the Simputer [14]. Simputer is a handheld running open source software. It has a Strong Arm SA-1100 RISC CPU with $32 \mathrm{MB}$ of DRAM. It runs Linux 2.4 and has a SIP-based VoIP client. We used a 3Com USB wireless adaptor to enable wireless connectivity on the Simputer.

Call setup: Figure 3 shows how an incoming and an outgoing call is setup. The Asterisk server on receiving any call request performs a table look-up to map a callee to his SIP address. After this caller-callee handshake, a VoIP connection is established between the clients. 


\begin{tabular}{|c|c|c|c|c|}
\hline Device (From/To) & PC & Simputer & Phone & Landline \\
\hline PC & Very good & Poor & Good & Very good \\
\hline Simputer & Average & Poor & Poor & Average \\
\hline Phone & Good & Average & Good & Very good \\
\hline Landline & Very good & Average & Very good & - \\
\hline
\end{tabular}

TABLE IV

VOIP QUALITY TEST RESULTS

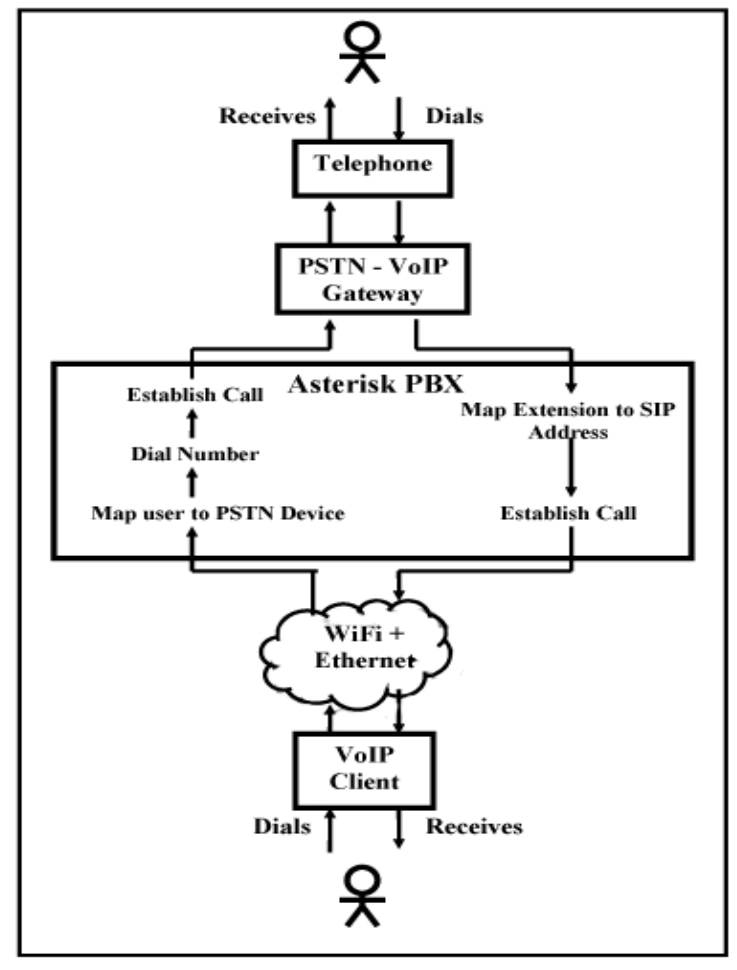

Fig. 3. Call flow

\section{EXPERIMENTAL RESULTS}

The system deployment architecture is shown in Figure 2(b). We evaluated the voice quality over different combinations of clients and at different locations. Calls between external landlines and the local devices (Simputer, analog phones and laptops) as well as intra-village calls were tested.

The quality of a VoIP call was defined based on its packet drop and delay characteristics as shown in Table III. Table IV summarises the voice quality for various configurations.

Calls to external PSTN numbers were of good quality except with the Simputer.

For intra-village connectivity, we found that we were able to do PC to PC calls quite easily over VoIP plus
WiFi. The quality was good and we had also used this mode of operation to do various tests about usable wireless ranges. We were able to do laptop to Simputer and vice versa too. The audio from Simputer to laptop was clear, but laptop to Simputer audio was quite poor. Simputer to Simputer voice calls were very difficult to do over WiFi. This was the case when two Simputers were connected through an AP, and also when both were connected directly in ad hoc mode.

\section{CONCLUSION AND FUTURE WORK}

We found that Ethernet is suitable for connecting the PSTN line to places that are nearby, with distances in the tens of metres. Beyond this, repeaters are required and the physical security of the cable is difficult to ensure. Cost is not a major factor here since Ethernet cable is relatively inexpensive. Further, voice quality over Ethernet was found to be very good.

We could span distances upto 150 metres using VoIP over WiFi. This method is also more scalable since it can serve a wider area. However the voice quality is not as good as with Ethernet. We believe this is partly due to codec problems on the Simputers we were using, and partly due to the nature of the wireless medium.

The system cost in general is determined by the cost of the hardware running the exchange, the gateway, the network infrastructure and the number of client devices deployed. We ran the software exchange (Asterisk) on a laptop. This has two constraints: i) the location of the software exchange is constrained by the lack of AC power outlets since we only had access to solar power; and ii) such a setup is not cost effective. We are currently building an integrated DC-powered device that combines the gateway and the software exchange. The device (shown in Figure 4) is an inexpensive motherboard with a PSTN gateway card. The motherboard runs the Asterisk server on a stripped-down version of Linux on flash memory. We expect this device to reduce the cost of deploying our architecture considerably. Currently the price of this system is about 8000 INR. While Simputers are not economical for use solely as a voice client, their 


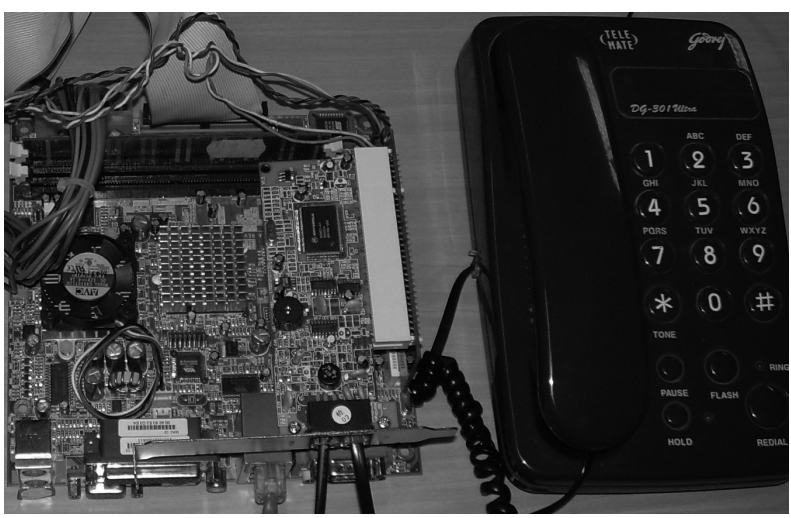

Fig. 4. Integrated exchange and gateway hardware

primary purpose was as learning devices for use in the school at Timbaktu. We expect the typical client devices to be low-cost VoIP phones.

Our proof-of-concept deployment in Timbaktu was for a small rural environment where twenty to thirty users have to share a single PSTN line. We expect our architecture to be suitable for other rural communities with similar requirements. Its applicability in larger areas is a subject for further study.

\section{ACKNOWLEDGMENTS}

We wish to thank everyone at the Timbaktu Collective for their support. In particular, we would like to mention Dr. U. Subbaraju and Subbu for their active help.

\section{REFERENCES}

[1] Consultation paper by TRAI, "Growth of telecom services in rural india," Downloadable from http://www.trai.gov.in/consultation.htm, October 2004.

[2] Department of Telecommunications, India, "Guidelines for Universal Service Support," Downloadable from http://www.dotindia.com/uso/usoindex.htm, 2005.

[3] DoT, India, "Village Public Telephone-Database and Monitoring System," http://www.moc.gov.in/report.asp, 2005.

[4] corDECT, "Wireless Access System," Technical Report, Midas Communication Technologies, Pvt. Ltd., 2000.

[5] S. Iyer, K. Paul, B. Ramamurthi, A. Kumar , "WiFiRe: A Rural Area Broadband Access System using the WiFi PHY and a New MAC," Technical Report, Centre for Excellence in Wireless Technologies (CEWiT), IIT Madras, Chennai, India, August 2006.

[6] The DGP MediaLab Asia Team, "Digital gangetic plains: 802.11-based low-cost networking for rural areas," http://www.iitk.ac.in/mladgp/, 2001-2004.

[7] LinkSys, "Linksys telephone adapter PAP2," available at http: //www.linksys.com, 2006.

[8] D-link, "D-link Antenna specification," available at http://www. dlink.com/products/?pid=53, 2005.

[9] Digium, "Asterisk opensource soft-pbx," available at http:// www.asterisk.org/about, 2005.

[10] CNA lab, "Asterisk setup and configuration files," available at http://www.it.iitb.ac.in/ cna/dokuwiki/doku.php?id= sravana:asteriskinstall, 2005.

[11] Sipura, "Telephone Gateway - SPA3000," available at http:// www.sipura.com/products/spa3000.htm, 2005.

[12] IETF-Real-time Applications and Infrastructure Area, "Session Initiation Protocol (sip)," http://www.ietf.org/html.charters/sipcharter.html, 2006.

[13] CNA lab, "Linksys pap2 setup and configuration files," available at http://www.it.iitb.ac.in/ cna/dokuwiki/doku.php? $i d=$ sravana:atainstall, 2005.

[14] Amida, "Amida simputer 4200," available at http://www. amidasimputer.com/, 2005. 